〈红外应用〉

\title{
远红外线的临床应用研究进展
}

\author{
李石林, 孙 悦, 潘华平, 丁成华, 袁丽霞, 石国栋, 李 琪, 李 洋 \\ (江西中医药大学, 江西 南昌 330004)
}

\begin{abstract}
摘要: 远红外线是一种热辐射, 对人类健康具有较好的生物效应。作为一种便捷无创的重要物理疗法, 远红外在疾病的诊断、治疗等临床领域中已得到广泛应用。本文收集了近年来远红外在医学临床应用 方面的相关研究文献, 对远红外的生物效应机制及其在疾病诊断、痛经、慢性疼痛、咳嗽、压疮、肿 瘤、腹泻等疾病治疗中的应用进行了总结, 为临床疾病的诊断和疗效评价提供参考依据, 以促进远红 外的临床应用与发展。
\end{abstract}

关键词: 远红外线; 生物效应; 疾病诊断; 临床治疗

中图分类号：TN219 文献标识码：A＼cjkstart文章编号：1001-8891(2020)09-0909-06

\section{Research Progress of the Clinical Application of Far Infrared Radiation}

\author{
LI Shilin, SUN Yue, PAN Huaping, DING Chenghua, YUAN Lixia, SHI Guodong, LI Qi， LI Yang \\ (Jiangxi University of Traditional Chinese Medicine, Nanchang 330004, China)
}

\begin{abstract}
Far infrared (FIR) is a form of thermal radiation that has beneficial biological effects on human health. As a convenient and non-invasive important type of physiotherapy, FIR has been widely used in clinical fields such as diagnosis and treatment of diseases. The author collected many related works in the literature regarding the clinical application of FIR to elaborate on its molecular mechanism and to summarize its application in diagnosis and clinical treatments of diseases including dysmenorrhea, chronic pain, cough, pressure ulcer, tumor, and diarrhea. This review provides a reference for the disease diagnosis and evaluation of curative effect to promote the clinical application and development of FIR.
\end{abstract}

Key words: far infrared, biological effect, disease diagnosis, clinical treatment

\section{0 引言}

红外线是太阳光线中不可见光线中的一种, 位于 光谱的可见光红光以外。在 20 世纪 80 年代中后期之 前, 远红外线包括太赫兹波（THz 波）, $\mathrm{THz}$ 波是指 波长在 $30 \mu \mathrm{m} \sim 1 \mathrm{~mm}$ 之间, 介于微波与红外之间的电 磁波。THz 波被正式命名之后, $\mathrm{THz}$ 波才与远红外线加 以区分。临床将红外线分为近红外线和远红外线, 医学 应用中的远红外波长介于 $4 \sim 400 \mu \mathrm{m}$ 之间, 可穿透 3 5 $\mathrm{mm}$ 的组织, 且对人体无辐射伤害。研究发现, 波长为 $4 \sim 15 \mu \mathrm{m}$ 的远红外线对人体的健康最为有益 ${ }^{[1]}$ 。目前, 临床应用中的远红外理疗设备可分为两大类 (图 1),
第一类是红外局部理疗仪 (图 1(a)), 理疗中较为普 遍的有红外灯、红外电子炎疗仪等, 该装置在电加热 时会发出远红外线辐射, 对局部区域进行照射。第二 类是红外全身理疗仪 (图 1(b)), 也被称为 “红外桑 拿房”, 该装置可全方位发出远红外线辐射, 对全身 进行照射。远红外（Far Infrared Ray, FIR）疗法属于 物理疗法的范畴, 已在临床中得到广泛的应用, 疗效 显著, 没有危害性。本文总结分析了远红外的生物效 应机制及其在疾病诊断与治疗中的应用概况, 以便为 今后远红外相关的临床应用、研究提供参考。

\section{1 远红外的生物效应机制}

收稿日期 : 2019-12-28; 修订日期 : 2020-06-29.

作者简介: 李石林, 男, 硕士研究生, 研究方向: 中医诊法理论与临床。E-mail:leeshilin168@sina.com。

通信作者: 潘华平 (1985-), 男, 讲师, 博士, 从事中西医结合、生物化学教学与研究。E-mail:panhp2004@163.com。 丁成华 (1957-), 女, 教授, 博士生导师, 从事中医诊法理论与临床研究。E-mail:chenghuading@163.com。

基金项目：江西中医药大学大学生创新创业训练计划（201910412017); 国家自然科学基金（81760842); 江西省教育厅科技项目（GJJ180680); 江西中 医药大学一流学科建设项目 (JXSYLXK-ZHYI030); 高性能柔性远红外线理疗仪的镇痛疗效研究 (JXSYLXK-ZHYI054) 
远红外的生物效应可分为热效应和非热效应, 热 效应可以有效促进人体的血液循环、改善新陈代谢, 非热效应可使蛋白质分子恢复正常形态结构和传递 生物能量。远红外辐射作用到人体细胞上的蛋白质和 小分子, 从而有效地使人体生物和组织恢复功能而达 到正常的状态 ${ }^{[2]}$ 。远红外辐射可能通过多种生物效应 机制发挥作用。迄今为止, 远红外的生物效应机制尚 未完全明确。当前, 研究相对较为明确的远红外生物 效应机制（图 2) 主要有: (1)FIR 增加内皮祖细胞 (Endothelial Progenitor Cells, EPC) 活性 (内皮祖细 胞是分化为内皮细胞的前体), 从而促进内皮前体细 胞生长, 有利于血管生成 ${ }^{[3]}$; (2)FIR 可上调血红素加 氧酶-1（H0-1），而 H0-1 将血红素裂解为胆红素和 一氧化碳, 两者均是有效的抗氧化剂, 可以改善氧化 还原状态并减少氧化应激 ${ }^{[3]}$; (3)FIR 通过增加外周血 流量引起层状切应力的增加, 从而刺激内皮一氧化氮 合成酶（eNOS）的表达, 上调 $\mathrm{eNOS}^{[4]}$; (4)FIR 直接

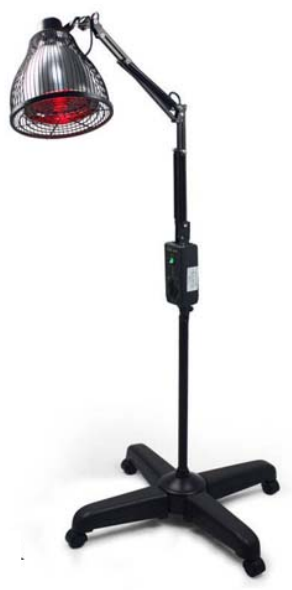

(a) 红外灯 (a) FIR lamp
上调 eNOS, 增加内皮细胞中一氧化氮 (NO) 的产生, 提高一氧化氮 (NO) 生物利用度, 从而改善内皮功 能 ${ }^{[5]}$; 5)FIR 上调核红细胞类似因子 $2(\mathrm{Nrf2})$ 的表达, 该转录因子可通过与细胞核中的内源性抗氧化剂响 应元件 (Anti-oxidative Response Element, ARE) 结 合来调节抗氧化剂的表达, 增强抗氧化作用, 从而提 高免疫力等 ${ }^{[6]}$ 。

此外, Jue-Long Wang ${ }^{[7]}$ 从细胞层面探寻远红外线 促进神经修复的机理, 实验发现 FIR 照射可以在培养 早期促进神经元样 PC12 细胞的神经突生长, 其作用 机制是激活 AKT1 磷酸化。Shanshan Shui ${ }^{[8]}$ 回顾和总 结以前的研究结果, 认为远红外治疗可能与内皮一氧 化氮合酶表达的增加以及一氧化氮的产生密切相关, 并且可能调节某些循环 miRNA 的表达。研究 ${ }^{[9]}$ 发现, 改善线粒体功能应该是 FIR 生物学效应的主要机制之 一, FIR 辐射上调了线粒体复合体 I 的活性, 然后增 加了 $\mathrm{NAD}+/ \mathrm{NADH}$ 的比例。

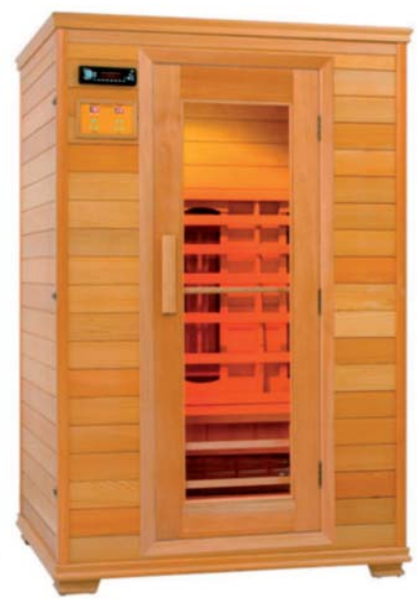

(b) 红外桑拿仪 (b) FIR suna

图 1 远红外理疗设备 Fig.1 Far infrared physiotherapy equipment

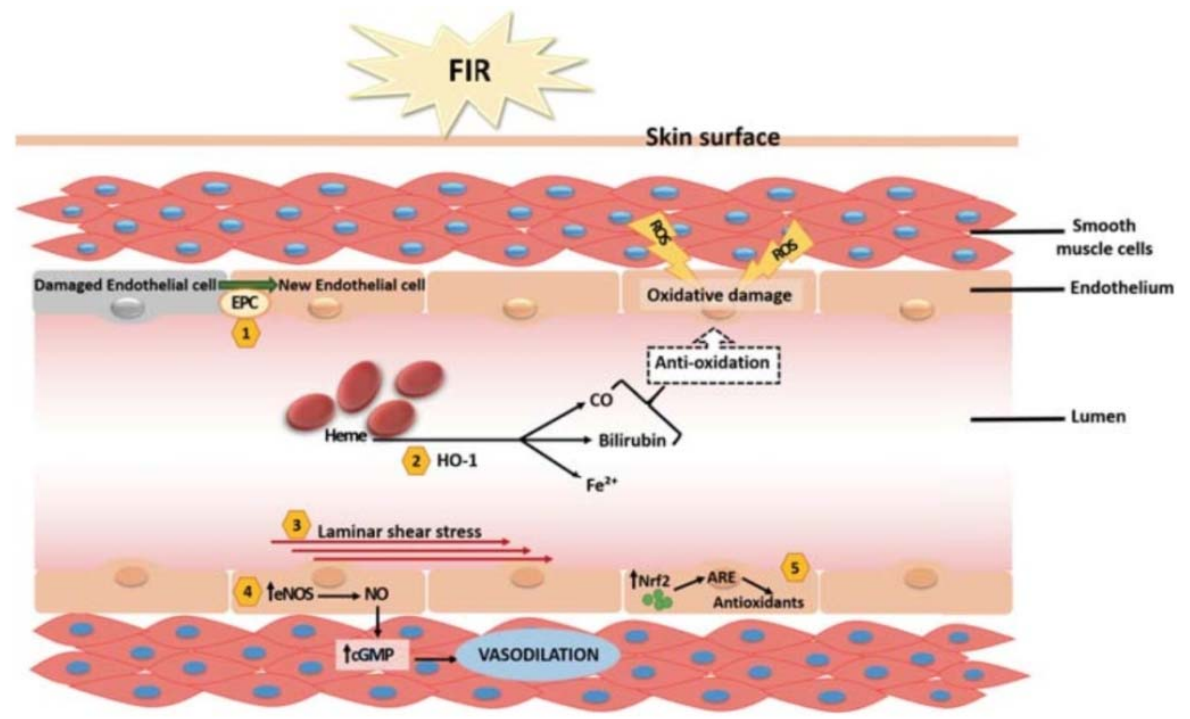

图 2 远红外生物效应机制 ${ }^{[3]}$

Fig.2 The mechanism of biological effects in far infrared ${ }^{[3]}$ 


\section{2 远红外线在诊断中的应用}

红外热成像可以反映出物体表面的温度场, 其参 数可间接地辅助疾病的诊断。程诚等 ${ }^{[10]}$ 使用医用远红 外热像仪采集缺血性脑血管病患者远红外热像图。与 健康体检者的头面部远红外热像图对比发现患者的 眶上区、内䀝区、额区、面规区温度差值明显大于健 康体检者。其中, 32 例患者行经皮血管内支架成型术 手术前后热像图温度发生变化, 各区域温度差值减 小。远红外热像图对缺血性脑血管病的诊断具有一定 的临床应用价值。罗芸等 ${ }^{[11]}$ 使用平面红外热像仪采集 急、慢性颈肩痛患者和健康体检者的全身及项背部远 红外热图, 发现颈肩痛组患者的远红外热图像左右存 在差异, 急性疼痛患者的疼痛区域温度差别较大, $2^{\circ} \mathrm{C}$ 以上; 而慢性痛痛患者疼痛部位的热像稍有差别, 以 低温改变为主。远红外热像检查可直接反映出急、慢 性期颈肩痛患者疼痛区域的温度波动特点, 为临床诊 治提供参考性意见。文献[12]报道了使用红外热像仪 测定舌面温度场, 通过研究生物产热计算出内部温度 等参数, 以此量化舌诊信息。因此, 远红外线在临床 疾病的诊断及治疗方面发挥着积极作用。

\section{3 远红外线在治疗中的应用}

远红外线的热效应和非热效应有着积极的生物 学效应, 可直接或间接的治疗疾病。远红外疗法隶属 于光疗, 是物理治疗的一种, 广泛应用于临床各科, 其作用方式包括远红外照射、远红外穴位敷贴以及各 式各样的远红外理疗器材。表 1 概括了远红外在临床 医学上应用的主要疾病、应用方法、检测参数以及作 用结果。

\section{1 在痛经治疗中的应用}

《素问・举痛论篇》云: “痛者, 寒气多也, 有 寒故痛也。”其又云: “寒气入经而稽迟, 泣而不行...... 客于脉中则气不通。” 因此寒邪容易导致经脉气血运 行不畅, 不通则痛。《素问・举痛论篇》云: “寒则 气收。” 故寒邪导致经脉、肌腠收缩而挛急。女子以 血为用, 寒邪易阻滞气血运行畅通, 故为妇科疾病常 见致病因素 ${ }^{[13]}$ 。王燕 ${ }^{[14]}$ 对 120 例中医辨证为寒凝血疼 型月经不调、痛经患者采用远红外理疗贴进行治疗, 月经不调患者贴敷后的其有效率为 $96.67 \%$, 痛经患者 贴敷后有效率为 $93.33 \%$, 疗效显著。文娜 ${ }^{[15]}$ 分别对 30 例痛经患者在神阙穴、关元穴、三阴交穴进行热敷 贴治疗, 有效率为 $86.7 \%$, 极大地减轻了患者的症状。 $\mathrm{Yu}-\mathrm{Min} \mathrm{Ke} \mathrm{K}^{[16]}$ 研究由远红外陶瓷材料制成的皮带对原 发性痛经患者的治疗效果, 测定使用前后腹部温度、
血流量以及经期疼痛的变化。临床纳入 51 名临床诊 断为原发性痛经的女性, 随后, 使用双盲方法将受试 者随机分配到 FIR 腰带组或对照腰带组。每个受试者 在腹部周围佩戴皮带 $30 \mathrm{~min}$ 。拆下皮带后, 进行腹部 温度和血流量的后处理测量。在月经期间整天在腹部 上系上安全带, 采用口头评定量表 (Verbal Rating Scale, VRS) 和数字评定量表 (Numerical Rating Scale, NRS）评估 3 个月经周期第 1 至 3 天的疼痛程度。结 果表明, 与对照组 (戴着假腰带) 相比, FIR 组 (戴 着 FIR 腰带) 的腹部温度升高了 $0.6^{\circ} \mathrm{C}$, 腹腔血流量 增加了 $3.27 \%$ 。FIR 组的口头评定量表和数字评定量 表得分均低于对照组。与空白组 (不戴安全带) 相比, FIR 组的痛经平均持续时间从 2.5 天减少到 1.8 天。FIR 腰带可有效缓解原发性痛经, FIR 腰带在缓解疼痛, 提高皮肤温度和促进血液循环方面提供了更好的治 疗效果, 且方便和易于使用, 并且不需要外部能源。

\section{2 在慢性疼痛及骨关节疾病治疗中的应用}

颈、肩、腰、腿痛是临床上常见的疾病。其病理 学基础是软组织损伤导致的无菌性炎症 ${ }^{[17]}$ 。《素问 - 举 痛论》对疼痛的论述, 病因在于 “寒”, 病机为 “不 通则痛” ; 从临床实践而论, 痛证为寒、热、内伤等 原因导致气血运行的缓慢或阻滞从而出现不通、不荣 之痛证 ${ }^{[18]}$ 。朱坤福 ${ }^{[19]}$ 采用东贝远红外磁疗贴 (颈椎病 型）诊疗颈型颈椎病患者, 在大椎穴、肩井穴和阿是 穴等穴位贴敷治疗贴, 总体有效率为 $88.67 \%$, 且其治 疗方法灵活, 患者可以自行敷贴, 简单实用。罗小亮 ${ }^{[20]}$ 使用远红外电热理疗仪治疗关节炎患者 50 例, 以疼 痛视觉模拟评分法的前后变化为疗效评价标准, 治疗 后试验组的疼痛视觉模拟评分低于对照组, 远红外电 热理疗仪对关节炎患者的疼痛有一定的减轻作用。潘 劲昭等 ${ }^{[21]}$ 探究红外线结合中药外敷患者腰部治疗腰 椎间盘突出症的临床疗效, 与单纯使用中药外敷做对 比, 发现红外线加中药外敷治疗腰椎间盘突出症有效 率明显高于对照组。韩铁军 ${ }^{[22]}$ 采用远红外炎热贴治疗 颈、肩、腰、膝痛以及慢性软组织损伤和痛经, 并与 采用阵痛尒治疗的对照组相对比, 总体有效率为 $92.8 \%$, 且受试患者中未出现不良反应。Yen-Ting $\mathrm{Lai}^{[23]}$ 评估远红外线 (Far Infrared Ray, FIR) 贴片减 轻患者肌筋膜疼痛综合征 (Myofascial Pain Syndrome, MPS）疼痛方面的功效, 使用视觉模拟量表来反应患 者疼痛的强度, 在治疗前后使用压力检测器 algometer 来测量压力疼痛阈值 $(\mathrm{P})$ 和最大疼痛耐受性 $(\mathrm{T})$ 。 结果显示, MPS 患者使用 24 小时 FIR 贴片可减轻疼 痛, 且女性患者的皮肤对 FIR 贴片更为敏感。

\section{3 在呼吸系统疾病治疗中的应用}


远红外线通过穴位作用于人体脏腑和经络, 可以 协调、平衡五脏六腑之间的关系 ${ }^{[24]}$ 。赵景岩 ${ }^{[25]}$ 探寻远 红外止咳贴对小儿毛细支气管炎的辅助治疗疗效, 对 照组 30 例患者进行常规抗病毒、雾化吸入治疗, 观 察组 30 例患者采用常规治疗结合穴位贴敷, 观察患 者的咳喘以及肺部啰音的变化, 结果显示观察组有效 率为 $93.33 \%$, 小儿喘息以及肺部啰音均显著减少, 疗 效优于对照组。李秀兰 ${ }^{[26]}$ 治疗 100 例小儿咳嗽, 对照 组患儿口服氨溴索联合扑尔敏肌肉注射, 观察组患儿 在对照组使用氨溴索和扑尔敏的基础上, 使用远红外 止咳贴进行穴位贴敷, 连续治疗 7 天, 判定疗效。其 中观察组效率为 $62.07 \%$, 优于对照组, 疗效显著, 操 作简单易行。

\section{4 在压疮治疗中的应用}

压疮是由于局部组织在长时间受压力与摩擦力 的作用下而发生持续缺血缺氧等情况, 从而造成组织 溃烂坏死 ${ }^{[27]}$, 好发于持续卧床、关节活动障碍、术后 和重症患者。张新萍等 ${ }^{[28]}$ 对 80 例危重患者使用自制 的远红外保护套, 结果表明其自制的远红外保护套能 有效防治组织损伤和 I 期压疮, 降低足部压疮发生的 概率。王科伟 ${ }^{[29]}$ 对 50 例压疮患者在常规护理基础上 采用远红外频谱照射, 患者的压疮治疗有效率为 $80 \%$, 效果优于对照组, 远红外频谱照射可以改善肌 肤组织血液循环, 干燥皮肤, 对促进压疮的愈合非常 有益。

\section{5 在肿瘤治疗中的应用}

在体温左右发射远红外线将有效抑制体内肿瘤 细胞的生长 ${ }^{[30]}$ 。乳腺癌术后上肢淋巴水肿是乳腺癌术 后常见的并发症, 远红外线可以有效地减少肢体流体 的体积和周长以及皮肤淋巴炎（DLA）的频率 ${ }^{[31]}$ 。但 关于远红外线的肿瘤学安全性和激活任何残留乳腺

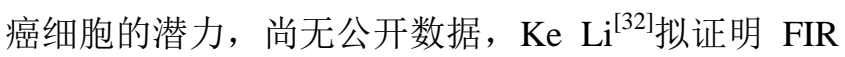
在临床和体外治疗 BCRL 方面的有效性和肿瘤学安全 性。纳入 5 年以上行乳房切除术并伴有上肢淋巴水肿 至少 1 年的患者, 随机分为观察组和对照组。观察组 采用远红外照射, 每天 $1 \mathrm{~h}$, 持续 4 周; 对照组采取 绷带包扎加压处理。1 年后随访, 检查静脉血中与乳 腺癌有关的 CA153 和 CA125, 超声检查肝, 脾, 肾, 乳房, 腋窝淋巴结, 锁骨上和锁骨下淋巴结是否有淋 巴结肿大。体外研究采用健康成年男子的皮肤二倍体 成纤维细胞和人乳腺癌细胞系 MCF-7 和 MDA-MB231 相对比, 在相同的培养条件下观察组行 远红外辐射, 对照组无辐射, 检测干预后细胞活力和 增殖率。结果显示: 观察组和对照组均未检测到 CA125 或 CA153 异常值的患者, 观察组与对照组中
CA125 和 CA153 值的变化相似, 观察组或对照组中 均未诊断出淋巴结肿大或新出现淋巴结肿大; 观察组 和未照射组之间的细胞活力和增殖均未显示明显差 异。确认远红外线治疗乳房切除术后淋巴水肿的肿瘤 学安全性。

\section{6 在腹泻治疗中的应用}

在神阙穴进行药物贴敷并配合远红外光波照射 可增加肠胃平滑肌张力, 促进胃肠蠕动, 从而改善胃 肠功能 ${ }^{[33]}$ 。李俊 ${ }^{[34]}$ 采用在口服蒙脱石口服液辅助远红 外穴位（神阙、中脘）敷贴治疗小儿腹泻, 结果显示 治疗后患儿的大便次数、大便性状恢复正常的时间均 小于单纯使用蒙脱石口服液。薛梅 ${ }^{[35]}$ 将远红外穴位贴 敷作为辅助治疗方式观察其对轮状病毒肠炎的临床 疗效, 常规基础治疗不变的情况下, 在神阙穴和中脘 穴使用含有远红外线粉的穴位贴，观察患儿呕吐及腹 泻次数。结果显示两到三天儿呕吐次数及腹泻次数较 对照组均减少。

\section{4 小结}

综上所述, 远红外不仅在疾病诊断中具有操作便 捷、成像直观、对人体无有害辐射等优点, 而且在多 种疾病的临床治疗中都有广泛应用，对人体有许多有 益的影响。然而，由于远红外的生物效应机制尚不完 全清楚, FIR 对生物的非热效应也有待明确, 其在医 学临床领域中的应用还不是很乐观。此外, 迄今为止 在体外细胞实验和动物中发现的远红外效应机制也 很少适用于人体，因此，远红外对人体的潜在生物效 应机制有待进一步研究。只有深入了解远红外对人体 的分子作用机制, 才能更好地普及 FIR 在临床领域中 的应用。如果可以证明非加热的 FIR 具有真实而显著 的生物学效应，那么 FIR 未来的应用前景更加广阔， 除了在医学领域, 在改善生活方式方面也有巨大的潜 在市场。在临床中, 远红外理疗仪器多为站立支架式, 使用不方便, 且效率低。因而为利用新技术、新材料, 研制高效、便捷的远红外理疗仪也颇为重要。研究与 利用远红外线成像诊断技术与纯净的远红外线对人 体的干预保健技术是未来红外医学的发展方向, 应用 前景广阔 ${ }^{[36]}$ 。随着材料学、生物学、医学等领域的不 断发展，相信远红外对人体分子作用机制的神秘面纱 一定会被逐渐揭开，FIR 及其相关产品在今后的临床 应用和改善人类生活方式等领域中也会扮演越来越 重要的角色。 


\section{参考文献:}

[1] 季冠芳, 杨子涁. 远红外线的生物学效应及其应用 [J]. 天津医药, 2007, 35(1): 78-80.

JI Guanfang, YANG Zibin. Biological effects of far-infrared rays and their applications[J]. Tianjin Medical Journal, 2007, 35(1): 78-80.

[2] 庞小峰. 生命体吸收的红外光的非热生物效应的研究 [J]. 物理, 2001, 50(9): 525-532.

PANG Xiaofeng. Study on the Non-thermal Biological Effect of Infrared Light Absorbed by Living Body[J]. Physics, 2001, 50(9): 525-532.

[3] Shemilt R, Bagabir H, LangC, et al. Potential mechanisms for the effects of far-infrared on the cardiovascular system - a review[J]. Vasa, 2019, 48(4): 303-312.

[4] Ziegler T, Silacci P, Harrison VJ, et al. Nitric Oxide Synthase Expression in Endothelial Cells Exposed to Mechanical Forces[J]. Hypertension, 1998, 32(2): 351-356.

[5] Förstermann U, Munzel T. Endothelial nitric oxide synthase in vascular disease: from marvel to menace[J]. Circulation, 2006, 113(13): $1708-1714$.

[6] Wakabayashi N, Slocum SL, Skoko JJ, et al. NRF2 talks, who's listening?[J]. Antioxid Redox Signal, 2010, 13(11): 1649-1663.

[7] WANG JL, LIN YC, YOUNG TH, et al. Far- infrared ray radiation promotes neurite outgrowth of neuron-like PC12 cells through AKT1 signaling[J]. J Formos Med Assoc. 2019, 118(2): 600-610.

[8] SHUI S, WANG X, CHIANG JY, et al. Far-infrared therapy for cardiovascular, autoimmune, and other chronic health problems: A systematic review[J]. Exp Biol Med (Maywood). 2015, 240(10): 1257-1265.

[9] Hsu YH, Chen YW, Cheng CY, et al. Detecting the limits of the biological effects of far-infrared radiation on epithelial cells[J]. Sci Rep., 2019, 9(1): 115-86.

[10] 程诚, 高宇红, 薛毅珑, 等. 远红外热成像在缺血性脑血管病中的应 用[J]. 中国医学影像学杂, 2014, 22(3): 210-212, 216.

CHENG Cheng, GAO Yuhong, XUE Yilong, et al. Application of far infrared thermographyin ischemic cerebrovascular disease[J]. Chinese Journal of Medical Imaging, 2014, 22(3): 210-212, 216.

[11] 罗芸, 薛毅珑, 高宇红, 等. 急慢性颈肩痛患者的远红外热像特征分 析 [J]. 中国激光医学杂志, 2008, 17(6): 431-433, 453.

LUO Yun, XUE Yilong, GAO Yuhong, et al. Far infrared thermal imaging characteristics of patients with acute and chronic neck and shoulder pain[J]. Chinese Journal of Laser Medicine \& Surgery, 2008, 17(6): 431-433, 453.

[12] 诸凯, 马一太. 中医舌诊中的生物传热问题研究概况 [J]. 上海中医药 杂志, 2003, 37(2): 58-61

ZHU Kai, MA Yitai. Review of Researches on Biological Heat Transfer in Tongue Diagnosis of Traditional Chinese Medicine[J]. Shanghai
Journal of Traditional Chinese Medicine, 2003, 37(2): 58-61.

[13] 练鹏颖, 周英. 论《傅青主女科》病因阐述 $[J]$. 辽宁中医药大学学报, 2018, 20(10): 187-190.

LIAN Pengying, ZHOU Ying. Explanation on the etiology of "Fu Qing Master and Daughter"[J]. Journal of Liaoning University of Traditional Chinese Medicine, 2018, 20(10): 187-190.

[14] 王燕, 王巨才, 王康俊. 远红外痛经理疗贴治疗妇科疾病 120 例的临 床观察[J]. 中国医疗器械信息, 2019, 25(5): 147-148.

WANG Yan, WANG Jucai, WANG Kangjun. Clinical observation of 120 cases of gynecological diseases treated by far infrared pain manager treatment tape [J]. China Medical Device Information, 2019, 25(5): 147-148.

[15] 文娜. 探讨远红外穴位热敷对妇科疾病之成效 [J]. 中国当代医 药,2009,16(24):153.

WEN Na.Discussion on the effect of far-infrared acupoint heat compress on gynecological diseases [J].China Contemporary Medicine, 2009,16(24):153.

[16] KE YM, OU MC, HO CK, et al. Effects of Somatothermal Far-Infrared Ray on Primary Dysmenorrhea: A Pilot Study[J]. Evidence Based Complementray \& Alternative Medicine, 2012, 2012(2): 240314.

[17] 宣哲人. 宣哲人软组织外科学 $[M]$. 上海: 文汇出版社, 2002: 3-295. XUAN Zheren. Xuan Zheren Soft Tissue Surgery[M]. Shanghai: Wenhui Publishing House, 2002: 3-295.

[18] 孙悦, 丁成华, 丁明, 等. 《素问. 举痛论》病因病机与诊治发微[J]. 世 界中医药, 2014, 9(3): 302-304.

SUN Yue, DING Chenghua, DING Ming, et al. The etiology, pathogenesis, and diagnosis and treatment of "Su Wen·Li Pao"[J]. World Chinese Medicine, 2014, 9(3): 302-304.

[19] 朱坤福, 祝蕾. 东贝远红外磁疗贴(颈椎病型)治疗颈型颈椎病疗效观 察[J/OL]. 临床检验杂志, 2018, 7(4): 762 .

ZHU Kunfu, ZHU Lei. Effective observation of Dongbei far-infrared magnetic therapy tape (cervical spondylosis type) in the treatment of cervical spondylosis of cervical type[J]. Journal of Clinical Laboratory Medicine, 2018, 7(4): 762.

[20] 罗小亮, 蒋中云. 远红外电热理疗仪治疗关节炎患者的有效性及安全 性[J]. 医疗装备, 2018, 31(14): 109-110.

LUO Xiaoliang, JIANG Zhongyun. Effectiveness and safety of far-infrared electrothermal physiotherapy in treating patients with arthritis[J/OL]. Medical Equipment, 2018, 31(14): 109-110.

[21] 潘劲昭, 韩转定, 鸿辉, 等. 红外线加中药外敷治疗腰椎间盘突出症 的临床效果分析[J]. 黑龙江医药, 2019, 32(1): 220-222.

PAN Jinzhao, HAN Zhuanding, HONG Hui,et al. Analysis of the clinical effect of infrared combined with external application of traditional Chinese medicine on lumbar disc herniation[J]. Heilongjiang Medicine Journal, 2019, 32(1): 220-222. 
[22] 韩铁军, 唐晓风, 陈钢. 远红外炎热贴临床应用 $[\mathrm{J}]$. 亚太传统医药, 2017, 13(12): 116-117.

HAN Tiejun, TANG Xiaofeng, CHEN Gang. Clinical application of far-infrared moxibustion hot paste[J]. Asia-Pacific Traditional Medicine, 2017, 13(12): 116-117.

[23] LAI Y T, CHAN H L, LIN S H, et al. Far-infrared ray patches relieve pain and improve skin sensitivity in myofascial pain syndrome: A double-blind randomized controlled study[J]. Complement Ther Med., 2017, 35: 127-132.

[24] 陈庆昭，陈家金金，邓新霞. 涌泉穴联合肺腧穴敷贴治疗小儿咳嗽 200 例临床观察 [J]. 中国民族民间医药, 2015, 24(7): 99-100.

CHEN Qingzhao, CHEN Jiaxin, DENG Xinxia. Clinical Observation on 200 Cases of Pediatric Cough Treated with Yongquan Acupoint Combined with Feiyao Acupoint Application[J]. Chinese Journal of Ethnomedicine and Ethnopharmacy, 2015, 24(7): 99-100.

[25] 赵景岩, 赵晶. 远红外止咳贴佐治毛细支气管炎疗效观察[J]. 临床合 理用药杂志, 2015, 8(5): 46-47.

ZHAO Jingyan, ZHAO Jing. Efficacy of far-infrared cough patch in treating bronchiolitis[J]. Journal of Clinical Rational Drug Use, 2015, 8(5): 46-47.

[26] 李秀兰. 远红外止咳贴治疗小儿咳嗽病疗效观察 [J]. 医学理论与实 践, 2014, 27(7): 892-893.

LI Xiulan. Efficacy of far-infrared cough patch in treating cough in children[J]. Medical Theory and Practice, 2014, 27(7): 892-893.

[27] 任文莉. 持续质量改进在医院护理管理中的实践应用及效果观察 [J]. 中国卫生产业, 2018, 15(10): 58-59 .

REN Wenli. Practical application and effect observation of continuous quality improvement in hospital nursing management[J]. China Health Industry, 2018, 15(10): 58-59.

[28] 张新萍, 徐建鸣, 于颖, 等. 预防压疮的远红外保护套制作与临床应 用[J]. 上海护理, 2016, 16(6): 62-64.

ZHANG Xinping, XU Jianming, YU Ying, et al. Production and clinical application of far-infrared protective sleeve for preventing pressure ulcers [J]. Shanghai Nursing, 2016, 16(6): 62-64.

[29] 王科伟, 谭洪栋. 远红外频谱照射治疗压疮的疗效观察 [J]. 中医临床 研究, 2018, 10(30): 89-91.
WANG Kewei, TAN Hongdong. Efficacy of far-infrared spectrum irradiation on pressure ulcers[J]. Clinical Journal of Chinese Medicine, 2018, 10(30): 89-91.

[30] Hamada Y, Teraoka F, Matsumoto T, et al. Effects of far infrared ray on Hela cells and WI-38 cells[J]. International Congress Series, 2003, 1255: 339-341.

[31] Hwang S, Lee DH, Lee IK, et al. Far-infrared radiation inhibits proliferation, migration, and angiogenesis of human umbilical veinendothelial cells by suppressing secretory clusterin levels[J]. Cancer Lett., 2014, 346(1): 74-83.

[32] LI K, XIA L, LIU NF, et al. Far infrared ray (FIR) therapy: An effective and oncological safe treatment modality for breast cancer related lymphedema[J]. J Photochem Photobiol B, 2017, 172: 95-101.

[33] 孙向宇, 龚正华. 中药神阙穴贴敷配合远红外照射治疗重症患者胃肠 功能障碍的疗效观察[J]. 中国中医急症, 2014, 23(12): 2307-2309.

SUN Xiangyu, GONG Zhenghua. Observation on the therapeutic effect of traditional Chinese medicine Shenqi acupoint application combined with far-infrared irradiation on gastrointestinal dysfunction in severe patients[J]. China Emergency, 2014, 23(12): 2307-2309.

[34] 李俊. 远红外穴位敷贴辅助蒙脱石口服液治疗小儿腹泻的疗效分析 [J]. 广州医药, 2018, 49(1): 33-35.

LI Jun. Efficacy analysis of far-infrared acupoint application assisted by montmorillonite oral solution in treating diarrhea in children[J]. Guangzhou Medical Journal, 2018, 49(1): 33-35.

[35] 薛梅. 远红外穴位敷贴佐治轮状病毒肠炎疗效评价 [J]. 辽宁中医药大 学学报, 2014, 16(8): 198-199.

XUE Mei. Efficacy evaluation of far-infrared acupoint application in treating rotavirus enteritis[J]. Journal of Liaoning University of Traditional Chinese Medicine, 2014, 16(8): 198-199.

[36] 李石林, 潘华平, 孙悦, 等. 穿戴式远红外理疗仪的研发与应用前景 分析 [C]//全国第十七届红外加热暨红外医学发展研讨会论文集, 2019: 206-207.

LI Shilin, PAN Huaping, SUN Yue,et al. Development and application prospect analysis of wearable far-infrared physiotherapy apparatus[C]//Proceedings of the 17th National Symposium on Infrared Heating and Infrared Medical Development, 2019: 206-207. 